\title{
Caregiver's Oral Healthcare Practices And The Level of Utilisation Of Oral Health Services and The Dental Caries Experience Of 3-12-Year-Olds Suffering From Heart Disease in Nairobi, Kenya
}

\author{
Daniel Kimei ${ }^{1}$ and Gladys N Opinya ${ }^{2 *}$ \\ ${ }^{1}$ BDS (Nairobi) MDS Pediatric Dentistry, Department of Pediatric Dentistry \& Orthodontics; School of Dental Sciences, College of the Health \\ Sciences University of Nairobi, Kenya
}

${ }^{2}$ BDS (Nairobi) CAGS, MSc. (Boston) Ph.D. Professor of Pediatric Dentistry, Department of Pediatric Dentistry \& Orthodontics; School of Dental Sciences; College of the Health Sciences University of Nairobi, Kenya

Received: 眥April 13, 2018; Published: 眥 April 26, 2018

*Corresponding author: Gladys Nabubwaya Opinya, Department of Paediatric Dentistry \&Orthodontics; School of Dental Sciences, College of Health Sciences, University of Nairobi, Kenya

\begin{abstract}
Cardiac diseases require that there is the meticulous maintenance of oral hygiene to avoid bacteremia, which has been associated with rheumatic heart disease and bacterial endocarditis. The aim was to establish the utilisation of oral health care and oral health practices of the caregiver about the oral hygiene and caries experience of children aged 3-12 years suffering from heart disease and were attending three pediatric cardiology clinics in Nairobi, Kenya. The study was descriptive and cross-sectional. It involved a study sample of children suffering from different types of cardiac conditions and attending the Pediatric cardiac clinics in three public institutions in Nairobi Kenya. The instruments the caregivers used to brush the children's teeth were the toothbrush $61(75 \%)$; chewing stick $14(17 \%)$ and $6(8 \%)$ never cleaned their teeth. Children who used a chewing stick had a lower dmft of $1.40 \pm 2.98$ compared to a dmft of $3.22 \pm 3.59$ among children who used the toothbrush, with Mann Whitney $\mathrm{U}, \mathrm{Z} \mathrm{p}=0.024$ ( $\mathrm{p} \leq 0.05)$. The children who brushed their teeth had a lower mean plaque score of $1.68 \pm 0.58$ compared those who did not clean with a mean plaque of $2.28 \pm 0.40$ with a Mann Whitney $\mathrm{U}, \mathrm{Z}=-2.611, \mathrm{p}=0.009(\mathrm{p} \leq 0.05)$. It was noted that the children who had visited a dentist had a higher caries experience with a dmft of $4.18 \pm 4.13$ and DMFT of $1.16 \pm 1.92$. However, the children who had never sought treatment at a dental facility had lower dmft of $1.89 \pm 2.88$; and DMFT of $0.36 \pm 1$, and the differences were statistically significant with Mann Whitney $\mathrm{U}, \mathrm{Z} \mathrm{p}=0.008(\mathrm{p} \leq 0.05)$. The plaque scores and caries experience were high in children whose caregivers had low aggregate utilisation of the oral health care facilities. However, those who had a low aggregate of oral hygiene practices had slightly higher plaque scores and caries experience.
\end{abstract}

Keywords: Cardiac Disease; Children; Utilisation; Oral Health Services; Caregivers

\section{Introduction}

Populations with chronic medical illness or other disabilities had the most unmet needs for oral health services [1], with poor oral hygiene and increased caries experience than the general population. For a child from a low-income family with heart disease, this means an added economic burden in an already tricky situation [2], as heart diseases necessitate regular dental check-ups and maintenance of meticulous oral hygiene. This concern has even been highlighted with new proposals on changes in the guidelines

relating to prophylaxis against infective endocarditis [3,4]. The oral conditions may have a considerable impact on the general health status and quality of life of otherwise healthy children, but their effects on those children with acute and chronic illness can be more dangerous [5]. Children with cardiac defects and diseases are at increased risk or even life-threatening complications [6]. Hence the need for preventive dental health care geared to reducing the risks associated with management of the oral conditions under general 
anaesthesia. Also, the prolonged bleeding from warfarin medication often taken By the children [7-10]. Poor oral hygiene may give rise to a frequent bacteraemia under normal physiological conditions, and this can lead to a permanent risk of developing heart disease [11-14]. Two common oral diseases, namely periodontal and dental caries, though preventable, are still more prevalent in Kenya $[15,16]$. The children with heart disease have the disadvantage that their caregivers are preoccupied with the with the primary medical condition the cardiac disease, resulting in the neglect of other facets of the child's total health [17]. The Kenya National Oral Health policy document has already indicated that the $\mathrm{dmft}$ value for Kenyan 5-year old children as at 2002 was $1.5 \pm 2$.2, while $43 \%$ of 6-8-year-old children had caries [15], underscoring the fact that caries is still very rampant amongst the child population in Kenya.

The study was descriptive and cross-sectional where all the patients aged 3 to 12 years and their caregivers attending paediatric cardiology clinics over a three month period at Kenyatta National Hospital (KNH), Gertrude's Garden Children's Hospital (GGCH) and Mater Hospital. A Purposive sampling had been used to select the study hospitals. Based on Kliegman. study, the study population sample was determined as 79 cases. However, 81 patients were recruited in the study. A semi-structured questionnaire was used to collect information on the socio-demographic characteristics of the children and the parent/guardian habits on oral health practices and utilization of oral health services. As children waited to consult the cardiologist clinical examinations done to record the oral health status. The examination was conducted using sterilized instruments and under natural daylight, with the participants seated on a chair facing the window. Great care was taken during periodontal probing for gingivitis, to avoid initiating bleeding that could lead to septicaemia as the children were not on prophylactic antibiotics. The results were recorded on predesigned individual questionnaire sheets, and a record of dental caries and plaque was done. The dental caries was then recorded as dmft for the primary dentition and DMFT in the permanent $[18,19]$, and the dental plaque was marked based on the Loe and Silness plaque score index [20]. Before commencement of the study, the examiner was calibrated by an experienced paediatric dentist on the collection of data relating to dental caries, and dental plaque Cohen's kappa index score of 0.87 and $0.85(n=10)$ was obtained for dental caries and plaque score respectively. The questionnaire was pre-tested before use. A duplicate clinical examination was also performed by the examiner to determine intra-examiner consistency, with results of Cohen's kappa index score of 0.91 and $0.86(n=12)$ being obtained for dental caries and plaque score respectively.

\section{Data analysis}

The data collected was cleaned, coded and analyzed using SPSS version 17-computer software from SPSS Inc. IL. The results obtained were compared and tested using Kruskal Wallis Chi-square and Mann Whitney U statistical tests, with statistical significance pegged at $95 \%$ confidence interval.

\section{Results}

The 81 children in the study, 44 (54.3\%) were males and 37 $(45.7 \%)$ females. Their ages ranged between 3-12 years with a mean age of 8.16 years $( \pm 2.81 \mathrm{SD})$, and the 6-9-year-olds accounted for the most substantial proportion of $33(40.7 \%)$ compared to the 3-5 year-olds who formed $16(19.8 \%)$. The differences in ages and gender were not statistically significant Chi $\chi 2=1.287$, two df, $p=0.525(p \leq 0.05)$. A total of $37(46 \%)$ children were from rural areas, $28(34 \%)$ were from Nairobi, and $16(20 \%)$ were from other urban centres other than Nairobi. The distribution of the children according to the type of heart disease, rheumatic (RHD) accounted for $36(44.5 \%)$ while infective endocarditis (IE) affected $4(4.9 \%)$. The duration since diagnosis of the cardiopathy ranged from less than one year to 12 years. Nearly half of the children, 40 (49\%) had been diagnosed with the disease for a duration of between 1 to 5 years, while those who had been diagnosed more than five years and those less than one year accounted for 30\% and $21 \%$ respectively. The caregivers' oral health care practices that included how the child's teeth were brushed; the frequency of brushing; and whether tooth brushing was supervised showed that 75(93\%) children cleaned their teeth and 6(7\%) children did not clean their teeth. Of the group that cleaned their teeth, 33(44\%) did it twice a day, $29(39 \%)$ once a day while $16 \%$ once in a while/occasionally. About supervision, 62 (83\%) reported cleaning their teeth without supervision while 13 were assisted by the caregivers. Inquiry on the ways the child's teeth were cleaned, 75\% (61) of the children used toothbrush and the rest of the results were as shown in Figure 1. The children who used toothpaste were 59 (79\%) while 16 (21\%) never use any toothpaste.

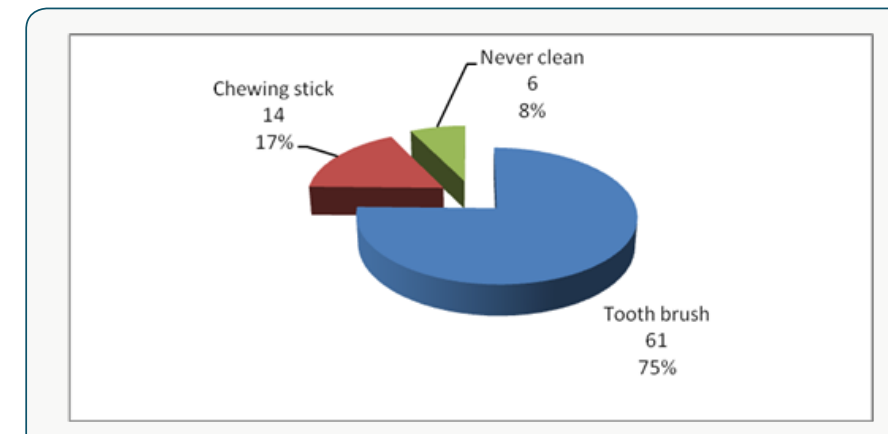

Figure 1: The different ways the children's teeth were cleaned.

Considering the utilisation of oral health care services by children with heart diseases; fifty-nine (72.8\%), children had never visited a dentist or utilised oral health services. Among the 22 (27.2\%) children who had been to a dentist, the dental procedure during the last appointment included extraction 10 (12.3\%). Also cleaning/prophylaxis (1(1.2\%)), consultation ; check-up 9(11.1\%) and fillings $2(2.5 \%)$.Caregiver's oral healthcare practices and the dental caries experience about the children five children who never cleaned their teeth had a higher dmft of $2.93 \pm 2.50$ compared to a lower dmft of $2.89 \pm 3.54$ among the 56 children who cleaned their teeth, and the differences were insignificant with $\mathrm{p}=0.957(\mathrm{p} \leq 0.05)$. 
The differences in the frequency of tooth cleaning, the eleven children who cleaned their teeth once in a while had a higher $\mathrm{dmft}$ of $3.36 \pm 5.29$ and the 23 children who cleaned twice a day had lower dmft of $2.68 \pm 2.77$, but.difference was not statistically significant with $\mathrm{p}=0.936(\mathrm{p} \leq 0.05)$. The children who used a chewing stick had a lower dmft of $1.40 \pm 2.98$ compared to a dmft of $3.22 \pm 3.59$ among the 46 children who used the toothbrush, with the difference was not statistically significant, $\mathrm{p}=0.024(\mathrm{p} \leq 0.05)$. The children who had visited the dentist apparently had a higher caries experience with dmft of $4.18 \pm 4.13$ and DMFT of $1.16 \pm 1.92$ when related to the children who had never visited a dentist, who had lower dmft of $1.89 \pm 2.88$; and DMFT of $0.36 \pm 1$. These differences in the results were statistically significant, $p=0.008(p \leq 0.05)$. The rest of the results are as shown in Table 1 . When the caregivers were classified into two groups based on the responses to the oral healthcare practices as being favourable or unfavourable practices,53 (86\%) caregivers fell in the unfavourable oral healthcare practices. Fiftythree children whose caregivers displayed unfavourable practices had a higher dmft of $3.62 \pm 3.54$ compared to $\mathrm{dmft}$ of $2.74 \pm 2.85$ among the eight children whose caregivers displayed favourable oral healthcare practices. The difference was statistically significant with Mann Whitney $U, Z=-1.297, p=0.197(p \leq 0.05)$. The mean plaque score was significantly lower among the 75 children who reported to cleaning their teeth with mean plaque scores of $1.68 \pm 0.58$, compared to a higher mean PS of $2.28 \pm 0.40$ among the six children who never cleaned their teeth with $p=0.009(p \leq 0.05)$. Those children who used the toothbrush had lower mean plaque scores of $1.64 \pm 0.61$. The children who cleaned more than twice a day had the lowest mean plaque score of $1.55 \pm 0.63$; and those who cleaned their teeth occasionally had the highest mean plaque scores of $1.99 \pm 0.41$, though these differences were not statistically significant with $\chi 2=0.067,1 \mathrm{df}, \mathrm{p}=0.936(\mathrm{p} \leq 0.05)$, Table 2 . The mean plaque scores among the $22(27 \%)$ children who had been to a dentist was mean PS of $1.68 \pm 0.55$ compared to higher plaque score of $1.83 \pm 0.61$ among the $59(73 \%)$ children who had never been to a dentist Table 2 . However, the difference was not significant, with $\mathrm{p}=0.422(\mathrm{p} \leq 0.05)$

Table 1: Relationship between caregivers' oral health practices and the dental caries experience.

\begin{tabular}{|c|c|c|}
\hline Oral Health Practice $s$ & dmft & Test of statistical significance $P \leq 0.05$ \\
\hline Yes $n=56$ & $2.89 \pm 3.54$ & \multirow{2}{*}{ Mann Whitney U Z= $-0.54: p=0.957$} \\
\hline No, $n=5$ & $2.93 \pm 2.5$ & \\
\hline Once a day $n=22$ & $2.87 \pm 2.77$ & \multirow{3}{*}{$\begin{array}{c}\text { Kruskal Wallis } \\
\text { Chi square }=0.007 \text {, d.f }=1, p=0.936\end{array}$} \\
\hline Twice a day $n=23$ & $2.68 \pm 3.33$ & \\
\hline Sometimes $\mathrm{n}=11$ & $3.36 \pm 5.29$ & \\
\hline \multicolumn{2}{|c|}{ Means of cleaning teeth } & \\
\hline Toothbrush $n=46$ & $3.22 \pm 3.59$ & \multirow{2}{*}{ Mann Whitney U, Z= $-2.251, \mathrm{p}=0.024$} \\
\hline Chewing stick $n=10$ & $1.40 \pm 2.98$ & \\
\hline \multicolumn{2}{|c|}{ Dental visits } & \\
\hline Yes $n=15$ & $4.73 \pm 4.08$ & \multirow{2}{*}{ Mann Whitney U Z= -2.641: $\mathrm{p}=0.008$} \\
\hline Never $n=46$ & $2.24 \pm 3.03$ & \\
\hline
\end{tabular}

Table 2: Relationship between oral hygiene practices and the oral hygiene levels.

\begin{tabular}{|c|c|c|}
\hline Practice & Plaque score & Test of statistical significance $P \leq 0.05$ \\
\hline Yes $n=75$ & $1.68 \pm 0.58$ & \multirow{2}{*}{ Mann Whitney U Z=-2.611:, p=0.009 } \\
\hline No $n=6$ & $2.28 \pm 0.40$ & \\
\hline Once a day $n=29$ & $1.66 \pm 0.53$ & \multirow{3}{*}{ Kruskal Wallis Chi square $=0.067$, d. $\mathrm{f}=1 \mathrm{p}=0.936$} \\
\hline Twice a day $n=33$ & $1.55 \pm 0.63$ & \\
\hline Sometimes $n=13$ & $1.99 \pm 0.41$ & \\
\hline \multicolumn{2}{|c|}{ Implements for cleaning teeth } & \\
\hline Toothbrush n=61 & $1.64 \pm 0.61$ & \multirow{2}{*}{ Mann Whitney U Z= $-0.966, p=0.334$} \\
\hline Chewing stick $n=14$ & $1.82 \pm 0.40$ & \\
\hline \multicolumn{2}{|c|}{ Dental visits } & \\
\hline Yes $n=22$ & $1.68 \pm 0.55$ & \multirow{2}{*}{ Mann Whitney U, Z=-0.895 ,p=0.422 } \\
\hline No $n=59$ & $1.83 \pm 0.61$ & \\
\hline
\end{tabular}

\section{Discussion}

Despite the majority of the respondents, 75(93\%), with the majority reporting that their children cleaned their teeth, only
$33(44 \%)$ of these children cleaned their teeth at least twice a day, 62(83\%), of them, cleaning their teeth without supervision by the caregivers. Seven children had never visited a dentist 
to have teeth cleaned teeth cleaned. Also, some children had occasional cleaning of their teeth, and this puts the children the risk of developing early childhood caries, gingivitis, and poor oral health. The poor oral health may which may give rise to frequent transient bacteremia during mastication or tooth brushing. Other studies among children with heart diseases have reported that 55 $\%$ of the children brushed their teeth twice a day [21,22] and that $46.1 \%$ of the children brushed three times a day. Owino et al [26] reported that $67.5 \%$ of the 12 -year-old children in a peri-urban area brushed their teeth. Franco et, al [25] in their study considered as disappointing the percentage of children with congenital heart disease who had never visited a dentist, a reflection of other results obtained in studies by Silva et al [23], Saunders et al.[18], and Fonseca et al [5]. In this study, the very high percentage of the children examined had never seen a dentist, with only 22(27.2\%) of the children have been to a dentist before the stu dy. Moreover, even though, most of the treatment, which had been offered during their visit to the dentist, was extraction, just as reported in a study, Ober et al [24]. The finding is alarming since the American Heart Association recommends that children with heart disease should visit a dentist for the institution of preventive measures.

The lower frequency of dental visits in this study compared to other studies in developed countries could be because of the reasons that include the fact that; most of the caregivers are ignorant on the importance of preventive dental care among the children with heart disease. Most of the patients examined were of lower socioeconomic status, therefore, could not afford the treatment. Also; the dental facilities in Kenya are limited, inaccessible and most of them lack skilled dental personnel who are well trained to offer treatment to children with special needs. The use of other tooth cleaning devices like the chewing stick was illustrated in this study. Majority of the children who were using this device were mostly from rural areas where other tooth cleaning aids may not be available. The outstanding fact was that the children examined were from different residential backgrounds. The patients who used the chewing stick in this study had significantly lower dental caries experience than those who used the toothbrush. The low caries experience in the children who used the chewing stick may be because they could not afford the snacks between meals. The low could probably be explained by the fact most of the children who used the chewing stick were from rural areas where the dental caries experience was shown to be lower compared to urban centres possibly because of the difference in the diet. Also, some studies have demonstrated the cariostatic and bacteriostatic properties of some specific species of trees, which are used as chewing sticks. It is also possible that a few children who started to use the brush late in life after severe early childhood caries had been established could have skewed the high caries experience illustrated among the children who were using the brush.

The caregivers' aggregate oral healthcare practices did not significantly influence the dental caries experience among the children in the present study. The lack of differences in the gadgets for cleaning the teeth may be due to the small sample size where there was a loss of statistical power. Fifty-three (65; 4\%) children whose caregivers were classified as portraying "unfavorable practices" had higher caries experience with mean dmft of $3.62 \pm 3.54(n=53)$ compared to $2.74 \pm 2.85(\mathrm{n}=8)$ among the children whose caregivers reported "unfavorable practices" on oral care. The children who had been to a dentist had a higher dmft than those children who had never been to a dentist. This finding illustrates that children visit a dentist when dental disease dental caries has already occurred and that the majority of the treatment offered was curative to relieve the symptoms, with little or no emphasis on preventive oral care. The lack of focus on preventive oral care was further illustrated by the high proportion of active, untreated caries component of dmft compared to filled or extracted teeth. Despite the fact that caregivers' aggregate oral health care practices had no significant relationship with the oral hygiene of the children as noted earlier, thirteen children whose caregivers reported "favourable practices" had lower plaque scores of $1.69 \pm 0.54$. However, the plaque scores of sixty-eight children whose caregiver's had reported favourable practices had a mean plaque score of $1.73 \pm 0.59$ slightly higher.The children who cleaned their teeth had significantly lower plaque scores compared to those children who never cleaned teeth. The children whose teeth were never cleaned were at high risk of developing sub acute bacterial endocarditis when compared to the children who cleaned teeth regularly. As during the tooth brushing process, there is the mechanical removal plaque thus reducing the possibility of increased bacterial colonization of the plaque and reducing chances of bacteraemia during mastication. It was noted the that toothbrushes were more effective in control of plaque compared to the use of chewing sticks, though there was no significant difference between the two groups. The results of these study showed that children who had been to a dentist displayed better oral hygiene than those children who had never been to a dentist, though there was no statistical difference. The difference perhaps indicates that the dentist visited previously could have offered oral hygiene instructions on good tooth brushing techniques. In addition to that, the caregivers' aggregate oral healthcare practices did not significantly influence dental caries experience among the children. Those children whose caregivers were classified as portraying "unfavorable practices "on oral care, had higher caries experience with mean dmft of $3.62 \pm 3.54(n=53)$ compared to $2.74 \pm 2.85(\mathrm{n}=8)$.

The children who had been to a dentist had higher dmft than those children who had never been to a dentist. The finding may be rationalised that children who visited the dentist they did so when dental caries had already occurred. The primary treatment offered was curative to relieve the symptoms, with little or no emphasis on preventive oral care. The situation was further illustrated by the high proportion of active, untreated caries component of $\mathrm{dmft}$ compared to filled or extracted teeth. 


\section{Conclusion}

The utilization of oral health care and oral health practices of the caregiver of the children was low, and only apparent used in case of emergency mainly. The oral hygiene, gingival index and dental caries experience in the study population was high.

\section{Study limitations}

The study was only for three months. Hence children who had had appointments in the previous clinics were excluded. The small sample size based in three cardiology clinics may have created a bias. The clinic was limited to 3-23-year-olds excluding the older children 13-17 this is the policy on how paediatric age cut off as defined by the ministry of health.

\section{Acknowledgment}

We thank Professor Loice Gathece for contribution in the design of the study. The Kenyatta National Hospital and the University of Nairobi Ethics and Research Committee fors approval of the proposal. Alice Lakati who helped in statistical work and Dr. E. Kagereki and Dr. Kiprop for data entry. The Nurses and the staff at the Paediatric Cardiac clinics at the KNH, Mater Hospital and the Gertrudes' Garden children Hospital for facilitating data collection during the clinical examinations for the patients. We acknowledge all the parents and children who participated in the study without whom the study would not have been a success.

\section{References}

1. Rosenkranz ER (1993) Surgery for congenital heart disease. Current Opinion in Cardiology 8: 262-275.

2. Berger ENH (1978) Attitudes and preventive dental health behaviour in children with the congenital cardiac disease. Australian Dental Journal 12: 87-90.

3. Balmer R, Frances AB (2003) The experiences with oral health and dental prevention of children with congenital heart disease. Cambridge Journals 13(5): 439-443.

4. Fitzgerald K, Fleming P, Franklin O (2010) Dental management for children with congenital heart disease. Primary dental care 17(1): 2125 .

5. Bayliss R, Clarke C, Oakley C, W Somerville, A G Whitfield, et al. (1983) The microbiology and pathogenesis of infective endocarditis. British Heart Journal 50(6): 513-519.

6. Fonseca MA, Evans E, Teske D, Thikkurissy S, Amini H (2009) The impact of oral health on the quality of life of young patients with congenital heart disease. Cardiol Young 19(3): 252-256.

7. Brown A (2009) caries prevalence and treatment needs for healthy and medically compromised children at a tertiary care institution in Saudi Arabia. Eastern Mediterranean health journal 15(2): 378-386.

8. Hobson P (1980) The treatment of medically handicapped children. Int Dent J 30(1): 6-13.
9. Court SD (1994) Report of the Committee on Child Health. Fit for the future. London: HMSO.

10. Moore RS, Hobsen P (1989) A classification of medically handicapping conditions and the health risks they present in the dental care of children. Cardiovascular, hematological respiratory disorders. Journal of Pediatric Dentistry 2: 73-78.

11. Hayes PA, Ferules J (2001) Dental screening of paediatric cardiac surgical patients. Journal of Dentistry for Children 68(4): 255- 258.

12. Smith AJ, Adams D (1993) The dental status and attitudes of patients at risk from infective endocarditis. British Dental Journal 174(2): 59-64.

13. (1991) Council on Dental Therapeutics: American Heart Association, Preventing bacterial endocarditis: a statement for the dental professional. Journal of the American Dental Association 122: 87-92.

14. Dajani AS, Taubert KA, Wilson W, Ann F. Bolger, Arnold Bayer, et al. (1997) Prevention of bacterial endocarditis - recommendations by the American Heart Association. Journal of the American Dental Association 96: 358-366.

15. Guntheroth WG (1984) How important are dental procedures as a cause of infective endocarditis? American Journal of Cardiology 54(7): 797801.

16. (2002) Kenya Ministry of Health. National Oral Health Policy and Strategic Plan.Government Printer. Nairobi.

17. Roberts GJ, Roberts IF (1981) Dental disease in chronically sick children. Journal of Dentistry for Children 48(5): 346-351.

18. Kliegman RM, Behrman RE, Jenson HB, Stanton BF. (2007) Nelson Textbook of Paediatrics. (18 ${ }^{\text {th }}$ edn) Philadelphia, PA: Saunders p.1953.

19. (1997) World health Organisation Oral Health Surveys: Basic Methods: ( $4^{\text {th }}$ edn).

20. Loe, silness (1964) plaque score index.

21. Dattani S, Hawley, GM Blinkhorn, AS (1997) Prevalence of dental caries in 12-13-year-old Kenyan children in urban and rural areas. Int. Dent J 47: $355-357$

22. Saunders CP, Roberts GJ (1997) Dental attitudes, knowledge, and health practices of parents of children with congenital heart disease. Archives of Disease in Childhood 76(6): 539-540.

23. Silva DB, Souza IPR, Cunha MC (2002) Knowledge, attitudes, and status of oral health in children at risk for infective endocarditis. International Journal of Pediatric Dentistry 12(2): 124-131.

24. Ober AO, Masiga MA, Kemoli AM (2006) oral health status and utilization of oral health service by children with cerebral palsy in Kenya. MDS thesis, University of Nairobi.

25. Franco E, Saunders CP, Roberts GJ, Suwanprasit A (1996) Dental disease, caries-related microflora and salivary IgA of children with severe congenital cardiac disease: an epidemiological and oral microbial survey. Pediatric Dentistry 18(3): 228-235.

26. Owino RO, Masiga MA, Ng'ang'a PM, Macigo FM (2007) dental caries and gingivitis among 12-year-old children in peri-urban Kitale, Trans-nzoia District. Thesis, University of Nairobi. 
(c) This work is licensed under Creative

To Submit Your Article Click Here:

Submit Article

DOI: 10.32474/MADOHC.2018.02.000129

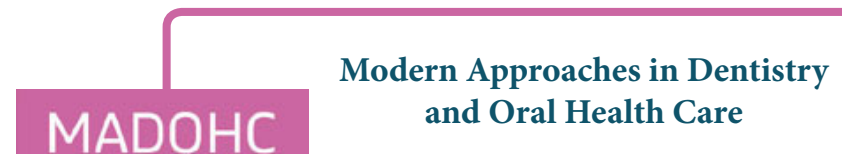

Assets of Publishing with us

- Global archiving of articles

- Immediate, unrestricted online access

- Rigorous Peer Review Process

- Authors Retain Copyrights

- Unique DOI for all articles 